\title{
Ionic Liquid Crosslinkers for Chiral Imprinted NanoGUMBOS
}

Suzana Hamdan, ${ }^{\mathbf{a}}$ Leonard Moore, Jr., ${ }^{\mathbf{a}, \dagger}$ Jason Lejeune, ${ }^{\mathbf{a}, \mathbf{f}}$ Farhana Hasan, ${ }^{\mathbf{a}}$ Trevor K. Carlisle, ${ }^{\mathbf{b}, \mathbf{f}}$ Jason E. Bara, ${ }^{\mathbf{b}, \overline{\mathbf{T}}}$ Douglas L. Gin, ${ }^{\mathbf{b}}$ Andrew L. LaFrate, ${ }^{\mathbf{b}, \mathbf{P}}$ Richard D. Noble, ${ }^{\mathbf{c}}$ David A. Spivak, ${ }^{\mathbf{a}, *}$ and Isiah M. Warner $^{\mathrm{a}}$

${ }^{a}$ Department of Chemistry, Louisiana State University, Baton Rouge, Louisiana 70803, US

${ }^{\mathbf{b}}$ Department of Chemistry \& Biochemistry, University of Colorado, Boulder, Colorado 80309, US

${ }^{\mathrm{c}}$ Department of Chemical \& Biological Engineering, University of Colorado, Boulder, Colorado 80309, US

\begin{abstract}
Molecularly imprinted polymers (MIPs) are an important class of selective materials for molecular specific sensors and separations. Molecular imprinting using non-covalent interactions in aqueous conditions still remains a difficult challenge due to interrupution of hydrogen-bonding or electrostatic interactions water. Newly developed crosslinking ionic liquids are demonstrated herein to overcome problems of synthesizing aqueous MIPs, adding to previous examples of ionic liquids used as monomers in non-aqueous conditions or used as MIP solvents. Vinylimidazole ionic liquid crosslinkers were synthesized and subsequently explored as matrix supports for fabrication of molecularly imprinted polymeric nanoGUMBOS (nanoparticles derived from a group of $\boldsymbol{u}$ niform $\boldsymbol{m}$ aterials $\boldsymbol{b}$ ased on organic salts). Each of the four crosslinkers incorporated a unique functional spacer between the vinylimidazole groups, and the performance of the corresponding molecularly imprinted polymers was evaluated using chiral recognition as the diagnostic. High uptake values for L-tryptophan were found in the 13-87 umol/g range; and chiral recognition was determined via binding ratios of L-tryptophan over D-tryptophan that ranged from 5:1 to 13:1 for polymers made using different crosslinkers. Not only are these materials good for chiral recognition, but the results highlight the utility of these materials for imprinting aqueous templates such as biological targets for theranostic agents.
\end{abstract}

Keywords: polymeric ionic liquids, nanoGUMBOS, aqueous molecular imprinting, chiral recognition, chiral amino acids, ionic liquid crosslinkers

ABBREVIATIONS: GUMBOS, group of uniform materials based on organic salts; NGMIPs, nanoGUMBOS molecularly imprinted polymers; L-Trp, L-tryptophan; MIP, molecularly imprinted polymer; TX-100, Triton X-100; NaAOT, sodium bis(2ethylhexyl) sulfosuccinate; D-Trp, D-tryptophan; AAPH, 2,2'-Azobis(2-methylpropionamidine) dihydrochloride; NMR, nuclear magnetic resonance; FTIR, Fourier transform infrared spectroscopy; TEM, transmission electron microscopy; [NTf2], bis(trifluoromethylsulfonyl)amide; NGNIPs, nanoGUMBOS non-imprinted polymers.

(C) 2015. This manuscript version is made available under the Elsevier user license http://www.elsevier.com/open-access/userlicense/1.0/ 
Present addresses

\section{Present Addresses:}

'Department of Chemistry, Grambling State University, Grambling, Louisiana 71245, US

${ }^{\ddagger}$ Environmental Health \& Safety, Louisiana State University, Baton Rouge, Louisiana 70803, US

${ }^{\mathfrak{E}}$ Membrane Technology and Research, Newark, California 94560, US

${ }^{\bar{T}}$ Department of Chemical \& Biological Engineering, University of Alabama, Tuscaloosa, Alabama 35487

${ }^{\mathbf{P}}$ Charas Scientific, Denver, Colorado 80239, US

*Corresponding author: Telephone: 225-5782868. Fax: 225-5783458.*E-mail: dspivak@1su.edu

\section{INTRODUCTION}

Ionic liquids (ILs) are molten salts which possess a range of relatively low melting points due to inefficient packing of bulky cations and small anions (or vice versa) [1],[2]. ILs show interesting physical and chemical properties such as low volatility, chemical stability, and high conductivity [1], [3]; and can be tailored by molecular design of either the anionic or cationic component for task-specific functions [4],[5]. An emerging class of solid phase materials based on tunable ionic liquid chemistry, collectively referred to as GUMBOS (group of uniform materials based on organic salts), has been demonstrated as capable of forming nanoparticles (nanoGUMBOS) with magnetic, fluorescent, $\mathrm{pH}$ sensitive, and anticancer properties [6-15]. To combine one or more of these properties into nanoparticle based theranostic agents, incorporation of a molecular recognition element using the method of molecular imprinting was investigated for formation of nanoGUMBOS molecularly imprinted polymers (referred to as NGMIPs) [16],[17].

Scheme 1 outlines the methodology envisioned for molecular imprinting of nanoGUMBOS, which begins with formation of a pre-polymer complex of interactive monomers with the target compound. The complex is subsequently polymerized to immobilize the positions of the interactive monomers within the surrounding polymer matrix. Removal of the template produces a shape selective binding cavity lined with interactive functional groups in a complementary array within the cavity [18]. An advantage of using polymerizable ionic liquids is the ability to perform the molecular imprinting process in aqueous media, which has only shown a few successes to date 
[19] and even fewer examples of chiral recognition [20-26] (arguably the most challenging test of molecular recognition).

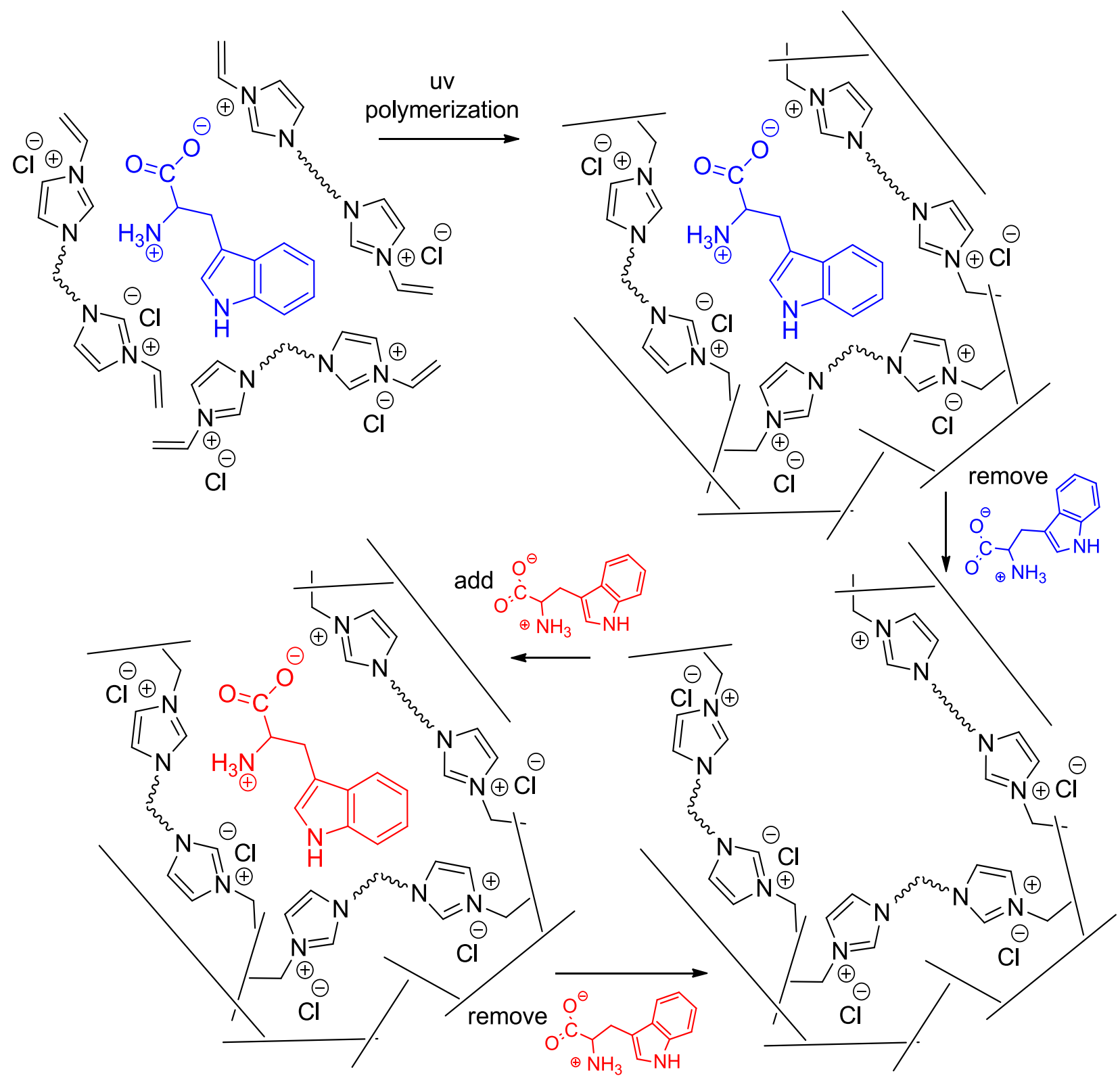

Scheme 1. Outline of the molecular imprinting method using crosslinking ionic liquids.

This study reports on the development of molecularly imprinted polymeric nanoGUMBOS directed toward molecular recognition of chiral amino acids using ionic liquid crosslinking 
monomers. A series of crosslinking ionic liquid monomers with different molecular spacers was synthesized [27-31] and used in this study for molecular imprinting with L-tryptophan (L-Trp) as the target compound. For this study, the ionic-liquid crosslinker was the only component of the final imprinted polymer (the template is removed), a strategy that has been shown to improve molecularly imprinted polymer (MIP) performance in systems that use crosslinkers that do not incorporate the ionic-liquid moieties.[32-34].To date, the utility of crosslinking ionic liquids for molecular imprinting has not been reported; however, imprinting with a non-ionic liquid crosslinking monomer has been shown to improve molecularly imprinted polymer (MIP) performance in other systems [32-34]. The enantiomer discrimination of chiral imprinted nanoGUMBOS synthesized with different crosslinkers was compared and correlated with the molecular structure of the crosslinkers.

\section{EXPERIMENTAL SECTION}

\section{Materials}

Ionic liquids and GUMBOS used in this study were received from the laboratories of Dr. Douglas Gin and Dr. Richard D. Noble at the University of Colorado, Boulder [27-31]. Triton X-100 (TX-100), sodium bis(2-ethylhexyl) sulfosuccinate (NaAOT), L- tryptophan (L-Trp), D-tryptophan (DTrp), as well as the initiator 2,2'-Azobis(2-methylpropionamidine) dihydrochloride (AAPH) were purchased from Sigma Aldrich (St. Louis, MO) and used as received. Ultrapure water $(18.2 \mathrm{M} \Omega \mathrm{cm})$ was obtained from an Elga Model model PURELAB ultra water filtration system.

\section{Methods}

Nuclear Magnetic Resonance (NMR) Spectroscopy. ${ }^{1} \mathrm{H}-\mathrm{NMR}$ experiments were performed by dissolving the polymerizable monomers in deuterated dimethylsulfoxide DMSO. The spectra (Fig. S1) were obtained using a Bruker AV-400 liquid instrument (Billerica, MA) that operates at 400 MHZ. For ${ }^{19} \mathrm{~F}-\mathrm{NMR}$, the studies on the monomers were performed on Bruker AVIII-400-Nanobay (AVB 400) instrument (Billerica, MA). 
Fourier Transform Infrared Spectroscopy (FTIR). The polymerized non-imprinted nanoGUMBOS which were suspended in an aqueous medium, were lyophilized. The formed pellet made of nanoparticles and surfactant was deposited on an ATR cell in a Bruker Tensor 27 instrument (Billerica, MA). The background signal of the surfactant was subtracted from the sample spectrum.

Transmission Electron Microscopy (TEM). TEM micrographs were obtained using a JEOL JEM-1011 TEM (München, Germany). An $8 \mu \mathrm{L}$ volume of sample was spotted onto ultrathin carbon coated 400mesh Ted Pella, Inc TEM grids (Redding, CA). The grids were mainly washed with water to avoid any unwanted adsorption of unpolymerized monomers and surfactant.

Fluorescence Spectroscopy. Fluorescence measurements were performed using a Fluorolog-3 fluorometer (HORIBA Scientific, Edison, NJ) at a right angle detection. The quartz sample cuvette was purchased from Starna Cells (Atascadero, CA) and had a $0.4 \mathrm{~cm}$ path length.

\section{Procedures}

Anion Exchange. The bis(trifluoromethylsulfonyl)amide ([NTf2]) anion of polymerizable monomers was exchanged with chloride $[\mathrm{Cl}]$ anion using an ion exchange resin. In a separation column, Dowex 1x8 chloride form (purchased from Sigma Aldrich) was packed and rinsed with $0.1 \mathrm{M}$ aqueous solution of sodium chloride. After flushing the column with deionized water, the monomers which were dissolved in 50:50 methanol:water (or acetonitrile:water for benzene monomer), were loaded on the column and eluted with water. The eluted samples were then spotted on a thin layer chromatography plate. Presence of the product was detected by appearance of a dark spot upon illumination with ultraviolet light. Water was removed by lyophilizing the samples. ${ }^{19} \mathrm{~F}-\mathrm{NMR}$ of the final product showed the disappearance of fluorine peak, which confirmed that ion exchange took place.

Synthesis of Non-Imprinted NanoGUMBOS Using UV-initiated Polymerization. For UV-initiated polymerization, $0.1 \mathrm{~mL}$ of monomeric aqueous solution $(0.1 \mathrm{M})$ and $5 \mathrm{~mL}$ of TX-100 aqueous surfactant solution $(0.02 \mathrm{M})$ were mixed under magnetic stirring. Ultimately, $0.2 \mathrm{~mL}$ of $0.005 \mathrm{M}$ aqueous AAPH solution was added to the mixture. After 30 minutes of magnetic stirring, the sample was purged with nitrogen and then exposed to UV light for 8 hours. 
Imprinting Procedure: Synthesis of the NGMIPs. To imprint the nanoGUMBOS with the chiral amino acid L-tryptophan, $0.26 \mathrm{mg}$ of L-tryptophan was added to the surfactant solution (described earlier) prior to UV-initiated polymerization. After polymerization, $1 \mathrm{~mL}$ of the polymerized sample was centrifuged for 30 minutes at $14000 \mathrm{rpm}$ speed (procedure performed in triplicate). To determine the theoretical number of binding sites created in the NGMIPs, samples were obtained from the supernatant and subsequent fluorescence measurement of supernatant gave the amount of free L-tryptophan that was not encapsulated into the polymeric matrices. The difference between the original amount of tryptophan and that found in the supernatant is recorded as the amount of tryptophan bound to the NGMIP sample, and represents the number of theoretical sites (maximum uptake) created inside the polymeric matrix (Fig. S2). To remove L-tryptophan from the cavities of synthesized nanoparticles, the NGMIPs were suspended in $1 \mathrm{~mL}$ of fresh water and dialyzed against deionized water using a Float-A-Lyzer obtained from spectrum laboratories (Irving, TX). The release of L-Trp was monitored by measuring the fluorescence of the dialysate over time (Fig. S3). The dialysis medium was changed initially each 4 hours and then each day for a period of two weeks.

Recognition Studies. After template removal, quantitative evaluation of tryptophan rebinding to the NGMIPs was performed by adding a solution of L- or D-tryptophan to the nanoGUMOS particles resuspended in water. An amount of tryptophan equal to the maximum uptake capacity (Fig. S2) was added to each aliquot of nanoGUMBOS suspensions (500 $\mu \mathrm{L}$ each). After vortexing followed by incubation for $20 \mathrm{hrs}$ on a shaker platform, the particles were removed by centrifugation and the supernatant analyzed by fluorescence to determine the amount of L- and D-tryptophan unbound. The measurements were compared to controls of L- and D-tryptophan representing the initial amount added to the nanoparticles. The amount of L- or D-tryptophan recognized is obtained by calculating the difference between the integrated surface areas of fluorescence spectra.

\section{RESULTS AND DISCUSSION}


Four different crosslinking ionic liquids distinguished by unique functional group spacers between the vinylimidazolium groups were investigated (Fig. 1) [27-31]. The different crosslinkers are referenced by the chemical structures as follows: benzene-based monomer (1), (PEG)-based monomer (2), alkane-based monomer (3), and alkyne-based monomer (4). The [NTf2] counterions of the synthesized crosslinker were exchanged with $\mathrm{Cl}^{-}$using an anion exchange column to render the compounds hydrophilic for aqueous imprinting [35]. The absence of the fluorine peak in

${ }^{19}$ F-NMR confirmed that NTf2 anions were fully exchanged with $\mathrm{Cl}^{-}$(Fig. S4).
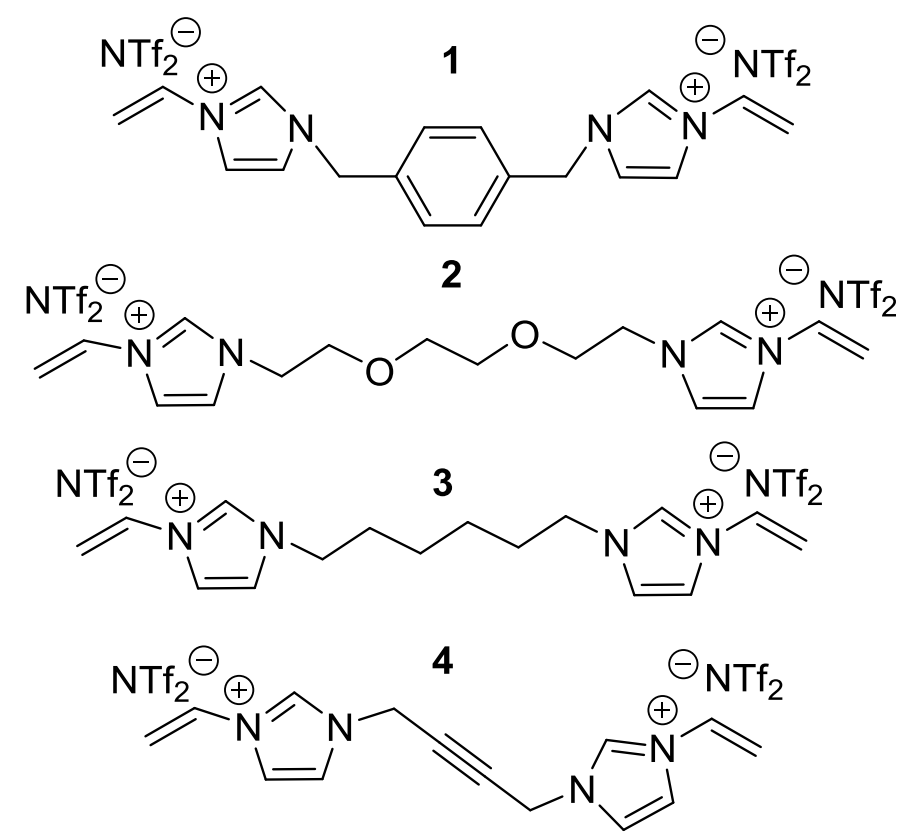

Fig. 1. Structures of polymerizable crosslinkers: (1) benzene-based monomer, (2) (PEG)-based monomer, (3) alkane-based monomer, and (4) alkyne-based monomer.

Before advancing to molecular imprinting, the synthesis of blank nanoGUMBOS (nanoGUMBOS non-imprinted polymers with the acronym NGNIP) in the absence of template was carried out using the four different crosslinkers. The NGNIPs were synthesized by dispersion polymerization of an aqueous solution using each of the four crosslinkers in the presence of Triton-X100 surfactant and 2,2'-Azobis(2-methylpropionamidine) dihydrochloride (AAPH) initiator under UV 
photoinitiation. TEM micrographs obtained after polymerization confirm the formation of nanoGUMBOS particles for each of the different crosslinkers (Fig. 2). As shown in TEM micrographs, the (PEG)- and alkyne-based polymerized NGNIPs had smaller average sizes of $87 \pm 10 \mathrm{~nm}$ and $44 \pm 5 \mathrm{~nm}$, respectively (Fig. 2b and 2d). The two other polymerized NGNIPs had average sizes of $109 \pm 16 \mathrm{~nm}$ for the alkanebased NGNIP and $118 \mathrm{~nm} \pm 23 \mathrm{~nm}$ for the benzene-based NGNIP. FTIR spectra were obtained before and after UV exposure of monomer

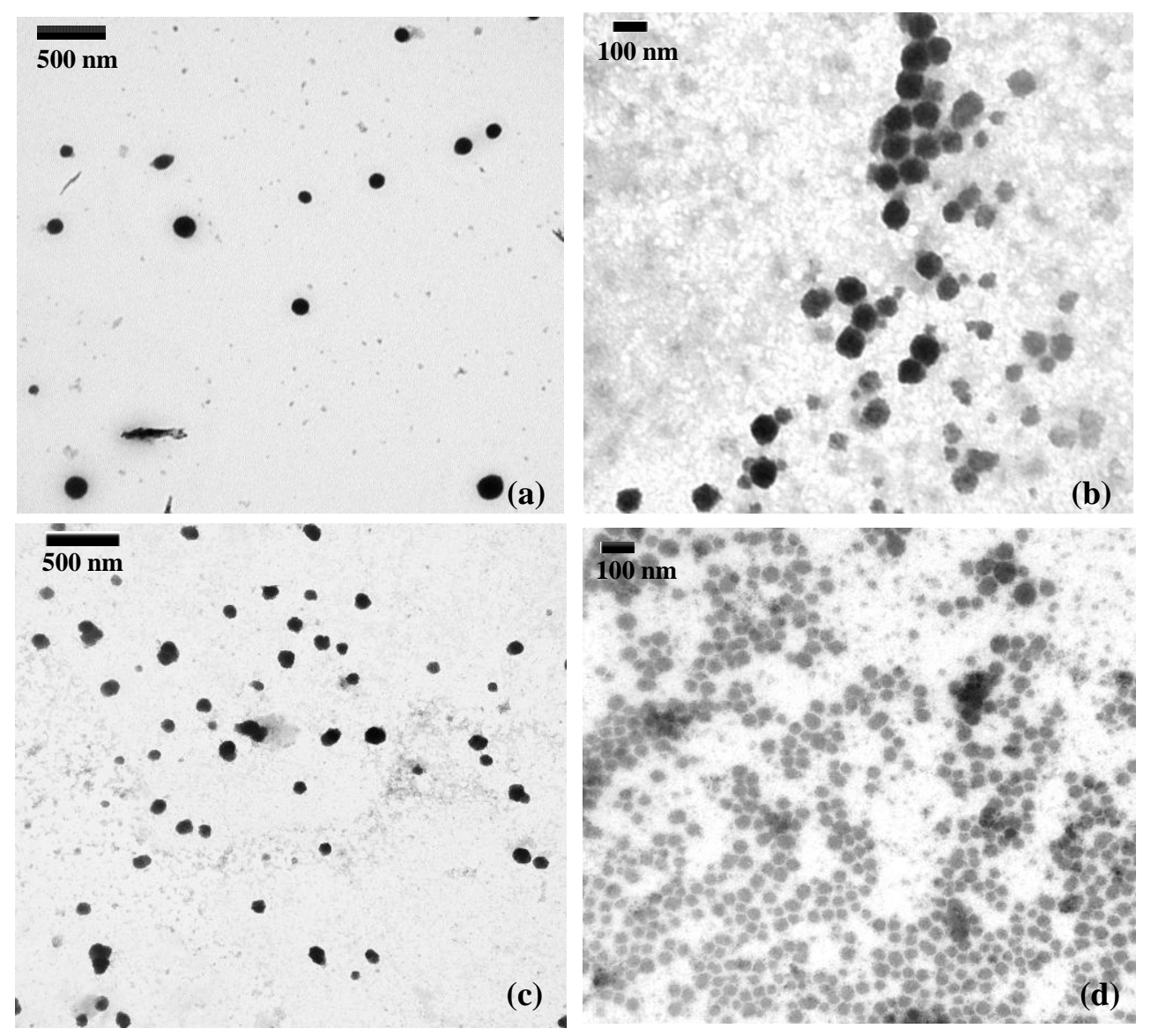

Fig. 2. Transmission electron micrographs of non-imprinted nanoGUMBOS (NGNIPs) polymerized with crosslinkers incorporating the following spacers: (a) benzene, (b) (PEG), (c) alkane, and (d) alkyne.

solutions. The surfactant peak was subtracted from the NGNIP spectra after the polymerization (Fig. 3). The decrease or disappearance of the vibration peak at $3080 \mathrm{~cm}^{-1}\left(=\mathrm{CH}_{2}\right.$ stretch vibration) compared to the standard peak at $1559 \mathrm{~cm}^{-1}$ confirms polymerization of the monomers [36]. Centrifugation of the 
polymerized samples gave a solid pellet, shown in the photograph in supplementary information (Fig. S5), which also confirmed polymerization.

Having established good protocols for the polymerization of NGNIPs, the ability to imprint in these materials with L-tryptophan was explored. It is known from the literature that the mole ratio of template to monomer is critical for eliciting the imprinting effect [37]. This is a result of
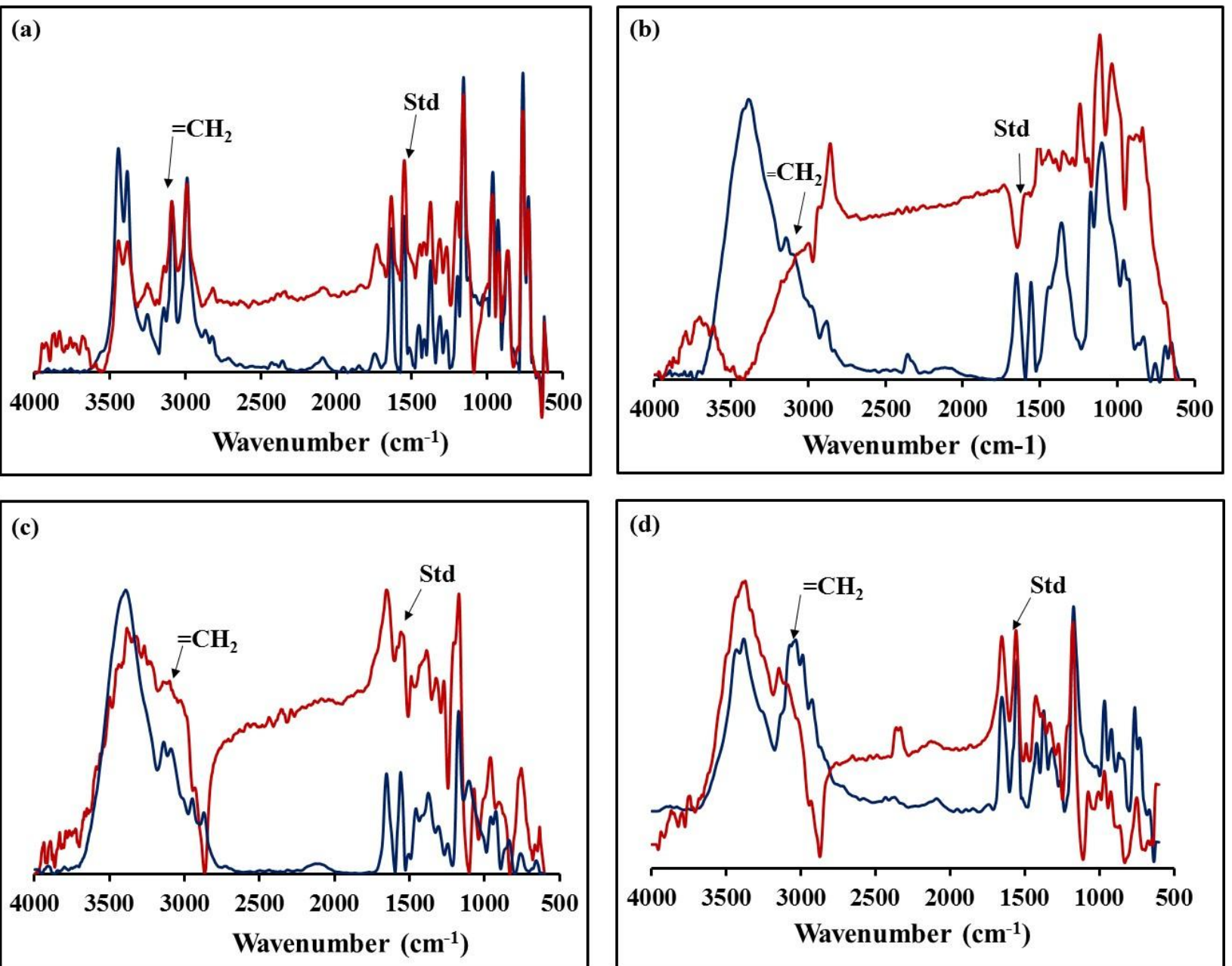

Fig. 3. FTIR spectra of (a) benzene-, (b) (PEG)-, (c) alkane-, (d) alkyne-based monomers and polymers (before and after irradiation respectively). The blue line represents the signal of monomers and the red line represents the signal of polymeric nanoparticles. The peak at $1559 \mathrm{~cm}^{-}$ ${ }^{1}$ is the standard peak which is used to compare the change in intensity of vibration peak at 3080 $\mathrm{cm}^{-1}$. 
balancing the number of binding sites arising from non-covalent pre-polymer complexes that increase with increased template concentration (via Le Châtelier's principle), versus the quality of binding sites that improves with a decrease of the template to monomer ratio. Preliminary studies on the NGMIPs using the (PEG)-based monomer were performed using the same formulation as the NGNIPs to compare template:monomer ratios of $1: 6$ and 1:8. After polymerization and centrifugation, analysis by fluorescence of the supernatant indicated that $29 \%$ of the L-Trp remained in the NGMIP with the template:monomer ratio $1: 8$, while $24 \%$ of the L-Trp remained in the particles formulated with a ratio 1:6 (Fig. S6). Thus, NGMIPs were synthesized using the 1:8 template:monomer ratio for all further studies. The amount of initiator and the time of photoinitiation were also optimized, arriving at a final procedure using $10 \mathrm{~mol} \%$ initiator and an 8 hour period of UV illumination with a Hanovia medium pressure mercury arc lamp. The optimized formulation and procedure were subsequently used to synthesize NGMIPs using each of the four different crosslinking ionic liquids. TEM micrographs of the NGMIPs provided in Fig. 4 show that average sizes of imprinted particles increased significantly versus the nonimprinted particles (Fig. 2) for the benzene-based NGMIPs (294 nm $\pm 46 \mathrm{~nm}$ ), PEG-based NGMIPs $(289 \pm 117 \mathrm{~nm})$, alkane-based NGMIPs $(196 \pm 37 \mathrm{~nm})$, and alkyne-based NGMIPs (205 $\pm 71 \mathrm{~nm}$ ) in Fig. 4a-d respectively. 


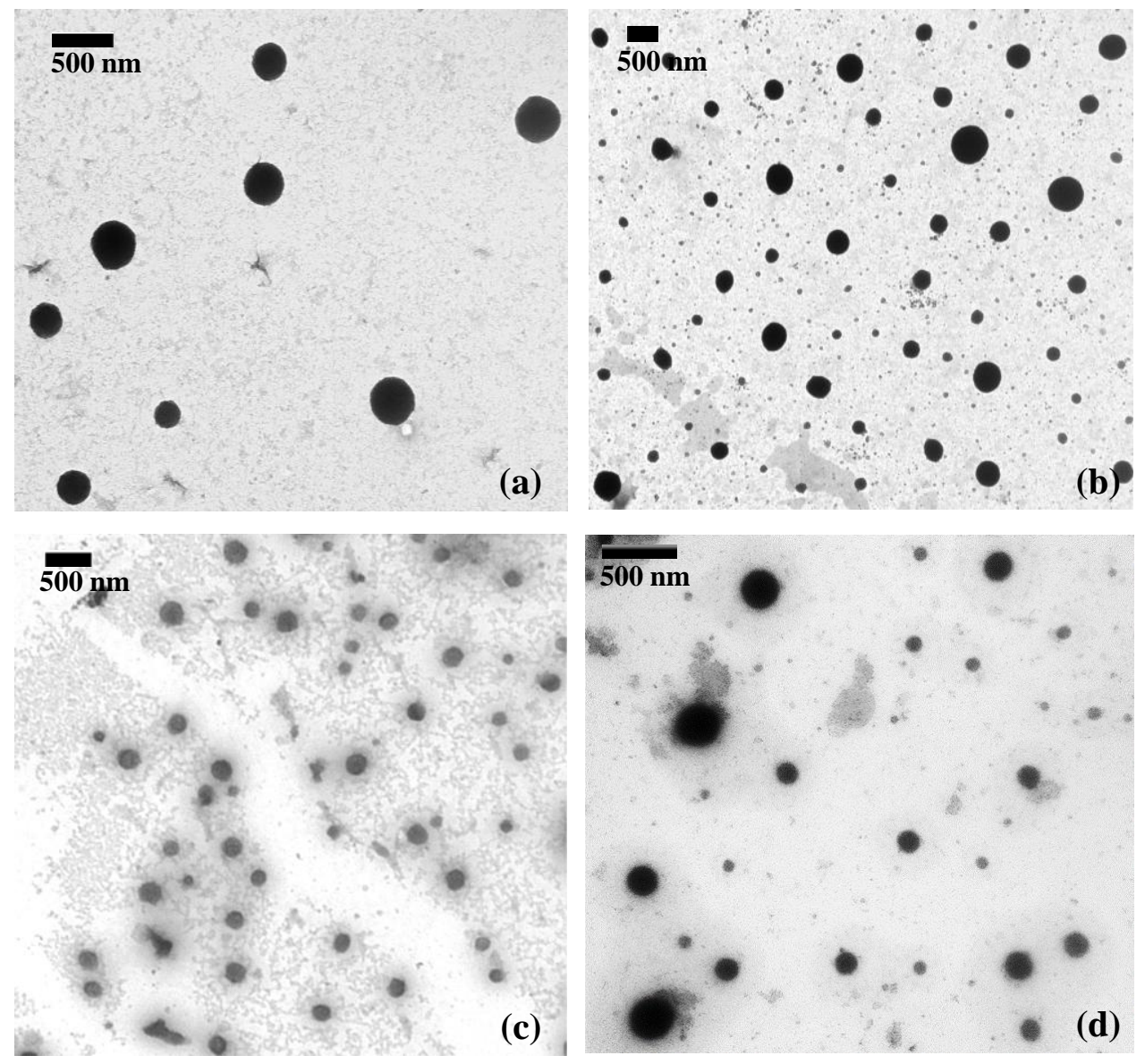

Fig. 4. Transmission electron micrographs of imprinted nanoGUMBOS (NGMIPs) polymerized with crosslinkers incorporating the following spacers: (a) benzene, (b) (PEG), (c) alkane, and (d) alkyne.

The increased sizes for the NGMIPs must be due to inclusion of the tryptophan template, which may be a result of changes in the surface tension characteristics from the equivalent polymers shown in Fig. 2. After polymerization and centrifugation, the supernatants of each of the NGMIPs were quantified by use of fluorescence spectroscopy to determine the amount of L-Trp that remained in each of the particles, and estimate the maximum uptake capacity of the respective NGMIPs (Fig. S2). Removal of the remaining L-Trp was carried out by dialysis over a two weeks period, until the dialysate showed no change in fluorescence (Fig. S3). Fig. 5 shows a TEM micrograph of the PEG-based NGMIPs after dialysis which appears to display a grainy structure that could result from tryptophan removal. The greater aggregation of the dialyzed NGMIPs 
versus the non-dialyzed NGMIPs (Fig. 4) is due to the removal of surfactant used in the initial imprinting of the particles.

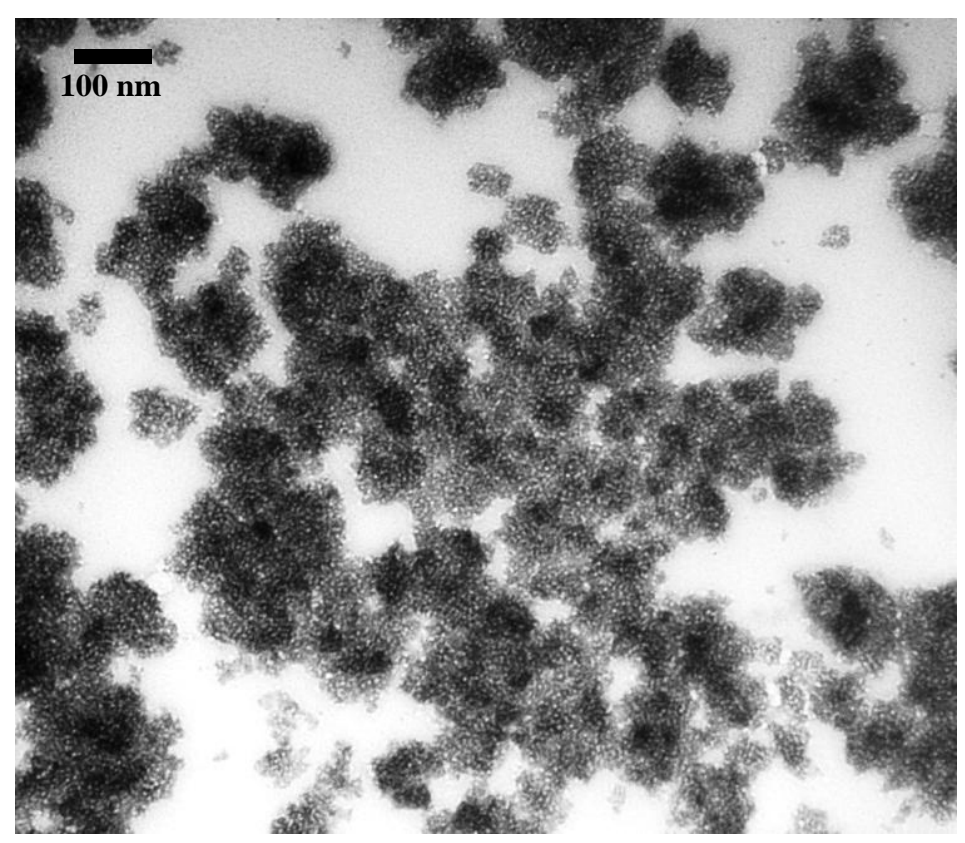

Fig. 5. TEM of the PEG-based NGMIP particles shown in Fig. $4 \mathrm{~b}$ after dialysis in deionized water for 2 weeks.

Rebinding of both enantiomers of tryptophan was analyzed in batch rebinding mode by adding to the NGMIP particles a solution of tryptophan with the concentration matching the maximum capacity determimed for each type of NGMIP (Fig. S2). After incubation with L- or D-Trp, the particles were removed by centrifugation and the amount of tryptophan remaining in the supernatant was measured by fluorescence (Fig. 6). The difference between the original solution concentration and the amount remaining in the supernatant gives the amount of tryptophan bound to the polymer, which is reported in Table 1 as the amount of tryptophan bound per gram of each NGMIP. The binding capacities of the NGMIPs were found to be in the range of $13 \mu$ moles/g $-87 \mu$ moles/g, which is approximately ten-fold higher in magnitude versus traditionally formed MIPs [38],[39]. Two factors that may be responsible for the high capacity of these materials is the strong binding of the cationic imidazolium group to the tryptophan 
molecule, and the large number of these interactions possible because two equivalents of imidazolium groups are incorporated for each monomer. The higher uptake value of the benzenebased nanomaterial (benzene-NGMIP) as compared to the other nanomaterials may be due to the pi-stacking and/or induced dipole interactions with the aromatic L-Trp that increases the affinity to these materials. This may also be true to some degree for the alkyne-NGMIP; however, the sizeable uptake by the alkyne-NGMIP is overshadowed by its poor selectivity (vide infra). The uptake by the alkane-NGMIP is a little lower due to the lack of pi-stacking interactions; and considerably lower for the (PEG)-NGMIP. The (PEG)-NGMIP is unique in this group of materials in the fact that it has glycol units that are hydrated by several water molecules per unit, which may disrupt some of the polar interactions between tryptophan and the binding site functionality in the (PEG)-NGMIP.

While binding affinity measured via the uptake by the NGMIPs is important, the greatest advantage of the NGMIPs, and all molecularly imprinted polymers as adsorbents or sensor materials, is the high level of molecular recognition possible for rebinding the template. Furthermore, the most challenging test of molecular recognition by any material is differential binding of enantiomers which differ only in their three dimensional configuration. In fact, enantiorecognition has long been known as the best indicator for testing the imprinting effect, 

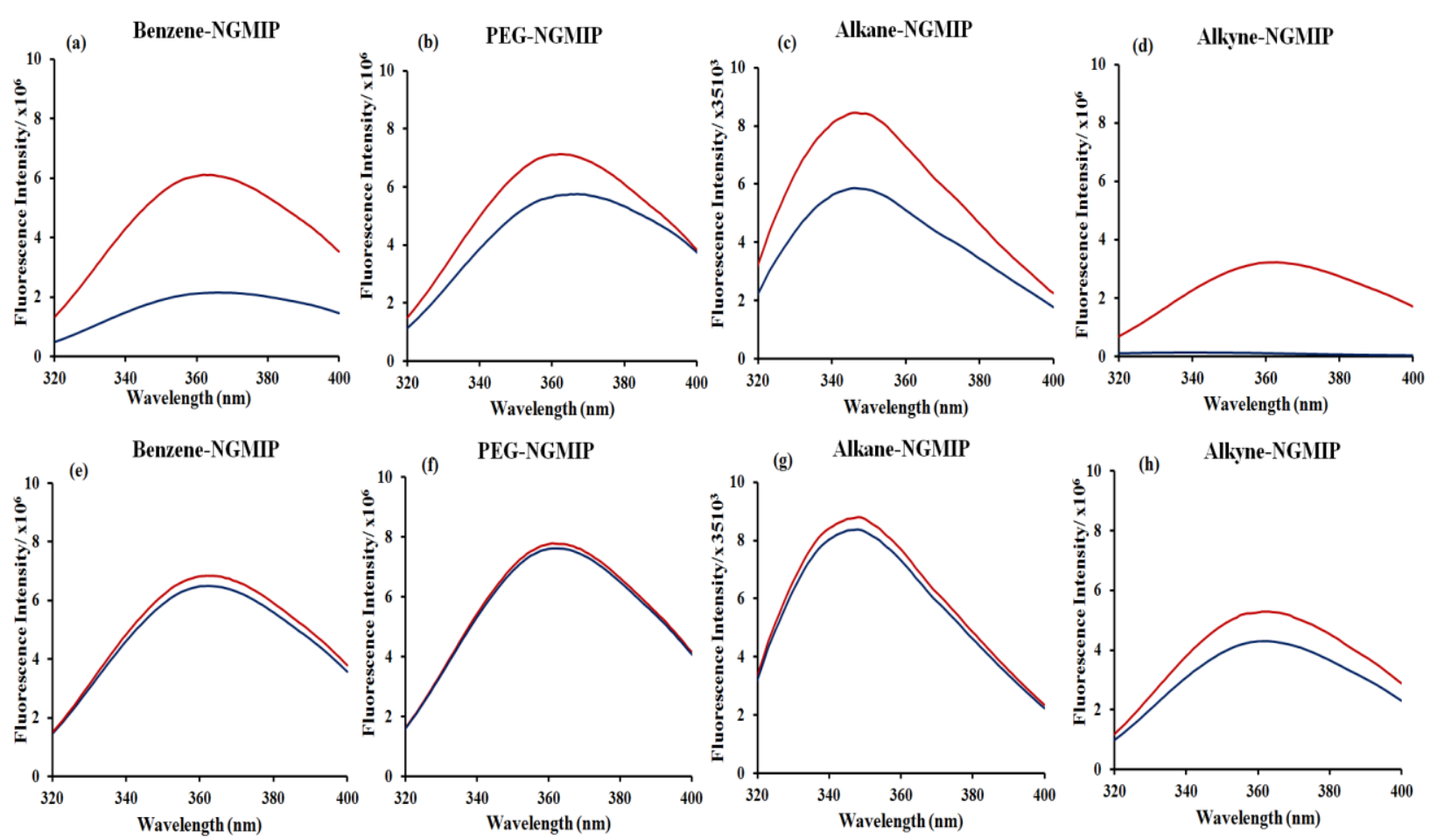

Fig. 6. Fluorescence spectra showing the relative amount of L-Trp (a-d) or D-Trp (e-h) rebound to the polymeric matrices. The red lines represent the amount of Trp in the incubation solution, and the blue line represents the amount of Trp left in the supernatant after the polymer with adsorbed Trp was removed. The difference between both spectra represents the amount of L-Trp rebound to the polymeric matrices, and the data are reported in Table 1 as an average over three trials.

because the specific binding in MIPs arises from complementary three-dimensional binding interactions. Therefore, enantiorecognition was evaluated for each of the NGMIPs by comparing the binding uptake of both L- and D-Trp on the L-Trp imprinted particles (Table 1). As shown in Table 1, all of the different NGMIP materials showed good chiral differentiation, with the benzene-NGMIP exhibiting the highest enantiomer binding ratio seen by the ratio of 13:1 for binding L-Trp over D-Trp. While the primary interactions of the crosslinkers with template are inferred to occur with imidazolium moiety, secondary interactions are postulated to exist between the aromatic spacer and the L-Trp template. The increased affinity and/or number of templatecrosslinker contacts would provide more complementary interactions that are specific to the L-Trp template 
Table 1. Comparison of the uptake and enantiomer binding ratio of each NGMIP.*

\begin{tabular}{ccccc}
\hline $\begin{array}{c}\text { Ionic Liquid } \\
\text { Crosslinker }\end{array}$ & $\begin{array}{c}\text { Benzene- } \\
\text { NGMIP }\end{array}$ & $\begin{array}{c}\text { (PEG)- } \\
\text { NGMIP }\end{array}$ & $\begin{array}{c}\text { Alkane- } \\
\text { NGMIP }\end{array}$ & $\begin{array}{c}\text { Alkyne- } \\
\text { NGMIP }\end{array}$ \\
\hline $\begin{array}{c}\text { L-Trp Rebinding } \\
\text { Uptake } \\
\text { (mmol/gram) }\end{array}$ & $0.087 \pm 0.029$ & $0.013 \pm 0.0038$ & $0.042 \pm 0.027$ & $0.081 \pm 0.0010$ \\
$\begin{array}{c}\text { D-Trp Rebinding } \\
\text { Uptake } \\
(\text { mmol/gram) }\end{array}$ & $0.0068 \pm 0.0054$ & $0.0019 \pm 0.0011$ & $0.0069 \pm 0.0007$ & $0.016 \pm 0.0055$ \\
$\begin{array}{c}\text { Enantiomer } \\
\text { binding ratio }\end{array}$ & $13: 1$ & $7: 1$ & $6: 1$ & $5: 1$ \\
\hline
\end{tabular}

*Error is reported as the standard deviation of the mean.

than the D enantiomer. This would account for the high chiral discrimination found for the benzene-NGMIP where secondary interactions arise from pi-stacking of the benzene spacer with the aromatic region of the L-Trp template. Enantiorecognition values are virtually the same for the other three NGMIPs, indicating that none of these spacer groups contribute significant interactions with the template (L-Trp) beyond the imidazolium-template contacts.

\section{CONCLUSIONS}

Four crosslinking ionic liquids were employed in the synthesis of novel nanomaterials that were successfully imprinted with the chiral template L-Trp under aqueous conditions. The fact that imprinting was performed in water is significant because in spite of many reports of rebinding analyses by MIPs in aqueous solutions, most of these examples do not perform the actual molecular imprinting process under aqueous conditions, but rather in organic solvents. The reason for this is that the non-covalent molecular imprinting technique relies on formation of pre-polymer complexes based on hydrogen-bonding and weak-to-moderate ionic interactions which are severely disrupted in the presence of water [19], [40-43]. 
However, many desirable templates, especially those of biological origin, are only soluble in aqueous matrices and cannot be adapted to organic soluble MIP methods. Thus, polymerizable ionic liquids are an important class of monomers for imprinting these aqueous-soluble targets. A few examples of molecularly imprinted polymers using non-crosslinking IL monomers have been published [44-49], and a nice example has been recently published using macromolecularly functionalized imidazole monomers [50]; however, there have not been any reports of chiral recognition using imprinted ionic liquids. Thus, a chiral template was imprinted for this purpose using crosslinking ILs developed by the groups of Gin and Noble that uniquely provide the necessary components to form nanoGUMBOS and simultaneously afford for the crosslinking required in the molecular imprinting process. Four different crosslinking ionic liquids were available with different functional groups as spacers between the vinylimidazolium groups, and the corresponding NGMIPs from each of these was compared. All four NGMIPs provided the first examples of enantiorecognition by molecularly imprinted polymeric ILs, indicating that multi-functional nanoGUMBOS with specific molecular recognition can be effectively synthesized for theranostic applications toward a spectrum of aqueous-based templates.

\section{ACKNOWLEDGMENT}

This material is based upon work supported by the National Science Foundation under Grant No. CHE1307611.

\section{REFERENCES}

[1] N. V. Plechkova, K. R. Seddon, Chem. Soc. Rev. 37 (2008) 123-150.

[2] M. G. Del Popolo, G. A. Voth, J. Phys. Chem. B 108 (2004) 1744.

[3] T. Welton, Chem. Rev. 99 (1999) 2071-2083.

[4] J. H. Davis, Chem. Lett. 33 (2004) 1072-1077.

[5] S. G. Lee, Chem. Commun. (2006), 1049-1063.

[6] D. K. Bwambok, B. El-Zahab, S. K. Challa, M. Li, L. Chandler, G. A. Baker, I. M. Warner, ACS Nano 3 (2009) 3854-3860

[7] A. Tesfai, B. El-Zahab, A. T. Kelley, M. Li, J. C. Garno, G. A. Baker, I. M. Warner, ACS Nano 3 (2009) 3244-3250.

[8] S. Das, D. Bwambok, B. El-Zahab, J. Monk, S. L. de Rooy, S. Challa, M. Li, F. R. Hung, G. A. Baker, I. M. Warner, Langmuir 26 (2010) 12867-12876.

[9] S. Das, S. L. de Rooy, A. N. Jordan, L. Chandler, I. I. Negulescu, B. El-Zahab, I. M. Warner, Langmuir 28 (2012), 757-765. 
[10] J. C. Dumke, B. El-Zahab, S. Challa, S. Das, L. Chandler, M. Tolocka, D. J. Hayes, I. M. Warner, Langmuir 26 (2010) 15599-15603.

[11] J. C. Dumke, A. Oureshi, S. Hamdan, B. El-Zahab, S. Das, D. J. Hayes, D. Boldor, K. Rupnik, I. M. Warner Appl. Spectrosc. 68 (2014) 340-352.

[12] A. N. Jordan, S. Das, N. Siraj, S. L. de Rooy, M. Li, B. El-Zahab, L. Chandler, G. A. Baker, I. M. Warner, Nanoscale 4 (2012) 5031-5038.

[13] S. Das, P. K. S. Magut, S. L. de Rooy, F. Hasan, I. M. Warner, RSC Adv. 3 (2013) 21054-21061.

[14] P. K. S. Magut, S. Das, V. E. Fernand, J. Losso, K. McDonough, B. M. Naylor, S. Aggarwal, I. M. Warner, J. Am. Chem. Soc. 135 (2013) 15873-15879.

[15] S. Hamdan, J.C. Dumke, B. El-Zahab, S. Das, D. Boldor, G. Baker, I.M. Warner, J. Colloid Interface Sci. , 446 (2015) 163-169.

[16] C. Alexander, H. S. Andersson, L. I. Andersson, R. J. Ansell, N. Kirsch, I. A. Nicholls, J. O'Mahony, M. J. Whitcombe, J. Mol. Recognit. 19 (2006) 106-180.

[17] L. Chen, S. Xu, J. Li, Chem. Soc. Rev. 40 (2011) 2922-2942.

[18] G. Wulff, Angew. Chem., Int. Ed. Eng. 34 (1995), 1812-1832.

[19] H. Zhang, Polymer 55 (2014) 699-714.

[20] O. Ramstrom, L. Ye, P. E. Gustavsson, Chromatographia 48 (1998) 197-202.

[21] Z. Jiang Y. Yu, H. Wu, J. Membr. Sci. 280 (2006) 876-882.

[22] Y. Zhang, Z. Pan, Y. Yuan, Z. Sun, J. Ma, G. Huang, F. Xing, J. Gao, Phys. Chem. Chem. Phys. 15 (2013) 17250-17256.

[23] M. Monier, A. El-Mekabaty, Int. J. Biol. Macromol. 55 (2013) 207-213.

[24] B. B. Prasad, A. Srivastava, I. Pandey, M. P. Tiwari, J. Chromatogr. B: Anal. Technol. Biomed. Life Sci. 912 (2013) 65-74.

[25] B. B. Prasad, I. Pandey, A. Srivastava, D. Kumar, M. P. Tiwari, Sens. Act. B-Chem. 176 (2013) 863-874.

[26] C-Y. Yue, G-S. Ding, F-J. Liu, A-N. Tang, J. Chrom. A. 1311 (2013) 176-182.

[27] T. K. Carlisle. Design, Synthesis, and Evaluation of New Ionic Liquid-Based Solvents, Polymers, and Composites for Enhanced Membrane based Carbon Dioxide/Light Gas Separations. University of Colorado at Boulder (2011).

[28] J. E. Bara. New Ionic Liquids and Ionic Liquid-Based Polymers and Liquid Crystals for Gas Separations. University of Colorado at Boulder (2007).

[29] T. K. Carlisle, G. D. Nicodemus, D. L. Gin, R. D. Noble, J. Membr. Sci. 397-398 (2012) 24-37.

[30] L. A. Robertson, M. R. Schenkel, B. R. Wiesenauer, D. L. Gin, Chem. Commun. 49 (2013) 9407-

9409.

[31] T. K. Carlisle, W. M. McDanel, M. G. Cowan, R. D. Noble, D. L. Gin, Chem. Mater. 26 (2014) 1294-1296.

[32] M. Sibrian-Vazquez, D. A. Spivak, Macromolecules 36 (2003) 5105-5113.

[33] M. Sibrian-Vazquez, D. A. Spivak, J. Org. Chem. 68 (2003) 9604-9611.

[34] J. LeJeune, D. A. Spivak, Anal. Bioanal. Chem. 389 (2007) 433-440.

[35] R. Marcilla, J. A. Blazquez, J. Rodriguez, J. A. Pomposo, D. Mecerreyes, J. Polym. Sci. A Polym. Chem. 42 (2004) 208-212.

[36] A. Svatos, A. B. Attygalle, Anal. Chem. 69 (1997) 1827-1836.

[37] D. A. Spivak, Adv. Drug Del. Rev. 57 (2005) 1779-1794.

[38] O. Kotrotsiou, S. Chaitidou, C. Kiparissides, Mat. Sci. Eng. C 29 (2009) 2141-2146.

[39] S. Chaitidou, O. Kotrotsiou, K. Kotti, O. Kammona, M. Bukhari, C. Kiparissides, Mat. Sci. Eng. B 152 (2008) 55-59.

[40] D. S. Janiak, P. Kofinas, Anal. Bioanal. Chem. 389 (2007) 399-404.

[41] T. Kubo, K. Hosoya, K. Otsuka, Anal. Sci. 30 (2014) 97-104.

[42] A. Murray, B. Ormeci, Environ. Sci. Pollut. Res. 19 (2012) 3820-3830.

[43] V. Pichon, F. Chapuis-Hugon, Anal. Chim. Acta 622 (2008) 48-61.

[44] L. Qian, X. Hu, P. Guan, B. Gao, J. Li, C. Wang, Y. Tang, Talanta 121 (2014) 56-64. 
[45] X. Luo, R. Dong, S. Luo, Y. Zhan, X. Tu, L. Yang, J. Appl. Polym. Sci. 127 (2013) 2884-2890.

[46] J. Fan, Z. Tian, S. Tong, X. Zhang, Y. Xie, R. Xu, Y. Qin, L. Li, J. Zhu, X. Ouyang, Food Chem. 141 (2013) 3578-3585.

[47] L. Guo, Q. Deng, G. Fang, W. Gao, W. Shuo, J. Chromatogr. A 1218 (2011) 6271-6277.

[48] W. Bi, M.Tian, K. H. Row, J. Chromatogr. A 1232 (2012) 37-42.

[49] X. Luo, Y. Zhan, X. Tu, Y. Huang, S. Luo, L. Yan, J. Chromatogr. A 1218 (2011) 1115-1121.

[50] L. Qian, X. Hu, P. Guan, D. Wang, J. Li, C. Du, R. Song, C. Wang, W. Song, Anal. Chim. Acta 884 (2015) 97-105. 


\section{Graphical Abstract}

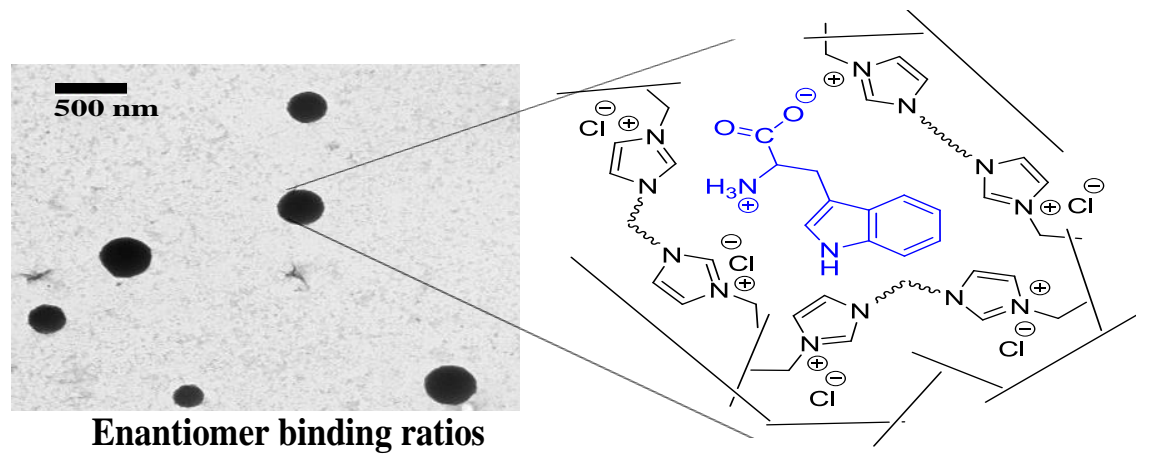

(L-Trp / D-Trp) = 5 - 13 